dies noch durch andere Maßnahmen flankiert werden, um keine Lücken entstehen zu lassen, die weiterhin widersinnige Ergebnisse ermöglichen würden. Ausgleichsmandate wären eine Lösung, würden den Bundestag aber vermutlich sehr stark aufblähen. Es empfiehlt sich daher, die Entstehung von Überhangmandaten soweit wie möglich von vornherein zu unterbinden. Dies könnte durch die Herabsetzung des Anteils der Direktmandate an der regulären Sitzzahl von derzeit 50 auf 40 oder 35 Prozent geschehen oder durch die Einführung von Zweimannwahlkreisen für die Wahl der Direktkandidaten mit der Erststimme. Die besondere Attraktivität letzterer Variante bestünde darin, dass sie den Charakter der personalisierten Verhältniswahl in vollem Umfang erhalten würde. Auch müsste die Anzahl der direkt gewählten Abgeordneten nicht gesenkt sondern könnte theoretisch sogar auf 60 Prozent erhöht werden, ohne dass die positiven Effekte verschwänden. Ein weiterer Vorteil der Zweimannwahlkreise läge darin, dass es damit mehr Parteien als bisher möglich wäre, mit direkt gewählten Kandidaten ins Parlament einzuziehen. Da jeder Wahlkreis durch zwei Abgeordnete vertreten wäre, würde die durchschnittliche ideologische Distanz der Bürger zu einem ihrer Wahlkreisabgeordneten sinken, was die gefühlte Repräsentation vermutlich erhöhen würde. In Zweimannwahlkreisen gäbe es einen wesentlich geringeren Anreiz zu strategischem Stimmensplitting, die Wähler hätten keine „Nachteile“ für ihre bevorzugte Koalition zu befürchten, wenn sie ihrer eigentlichen Präferenz entsprechend wählen.

Daher sollten Zweimannwahlkreise als interessante und vielversprechende Möglichkeit gesehen werden, die Probleme zu lösen, die sich durch die Überhangmandate im Generellen und den Urteilsspruch des Bundesverfassungsgerichts im Speziellen stellen. Der Aufwand des Neuzuschnitts der Wahlkreise wäre aller Voraussicht nach geringer als 2001 bei der Reduzierung der Wahlkreise von 328 auf 299 und dürfte den erwarteten Nutzen allemal wert sein.

\title{
Überhangmandate ohne negatives Stimmgewicht: Machbarkeit, Wirkungen, Beurteilung*
}

\author{
Franz Urban Pappi und Michael Herrmann
}

Der Deutsche Bundestag muss das Bundeswahlgesetz bis zum 30. Juni 2011 so geändert haben, dass kein negatives Stimmgewicht mehr auftritt. ${ }^{1}$ Dadurch, dass eine Partei in einem Bundesland mehr Direktmandate gewinnt, als ihr nach dem Zweitstimmenanteil zustehen, kann es vorkommen, dass eine zusätzliche Zweitstimme für diese Partei zu dem Verlust eines Mandats führt. Aus dem Überhangmandat kann so ein normal zustehendes Mandat in diesem Land werden. Das schadet der Partei in diesem Bundesland nicht, sie hat

* Wir bedanken uns für kritische Kommentare bei Ossip Fürnberg, Johann Hahlen und Eric Linhart, die zu zahlreichen Verbesserungen geführt haben, ohne dass die Genannten unsere Ansichten vollends teilen.

1 BVerfG 2 BvC 1/07 und 2 BvC 7/07, Beschluss vom 3. Juli 2008.

Zeitschrift für Parlamentsfragen (ZParl), Heft 2/2010, S. 260 - 278 
so oder so gleich viele Mandate. Der perverse Effekt kann vielmehr in einem anderen Bundesland, das heißt bei einer verbundenen Landesliste dieser Partei auftreten. Dadurch, dass im Land A aus einem Überhangmandat ein normal nach dem Zweitstimmenkontingent zustehendes Mandat wird - nehmen wir an, das letzte, das dieser Partei zusteht - verliert die Partei im Land B dieses Mandat, das sie ansonsten bekommen hätte. In der Literatur wird dieses Phänomen als negatives Stimmgewicht ${ }^{2}$ oder als inverser Erfolgswert ${ }^{3}$ bezeichnet.

Findet die Bundestagswahl in allen Wahlkreisen am selben Tag statt, was der Normalfall ist, fällt das mögliche negative Stimmgewicht nicht weiter auf. Dazu bedarf es einer kontrafaktischen Überlegung nach dem Motto: „Hätte die CDU in Thüringen 4.000 Stimmen weniger erhalten, wäre ihr Abrundungsmandat nach Hessen gewandert (...). “4 Erst die durch den Tod der NPD-Kandidatin im Wahlkreis Dresden I notwendig gewordene Nachwahl 14 Tage nach der Bundestagswahl vom 18. September 2005 machte das negative Stimmgewicht einer breiteren Öffentlichkeit bewusst. So führte zum Beispiel Martin Fehndrich am 23. beziehungsweise 26. September 2005 auf www.wahlrecht.de in einer Beispielrechnung vor, dass mindestens 42.000 CDU-Zweitstimmen in Dresden I zu dem Verlust eines Listenmandats in Nordrhein-Westfalen führen würden. Tatsächlich wählten dann bei 57.931 Erststimmen nur 38.208 Dresdner die CDU mit der Zweitstimme, was die Aufteilung der Zweitstimmensitze auf die Landeslisten der CDU (Abrundungsmandate nach Meyer) im Vergleich zum vorläufigen Wahlergebnis ohne Dresden I unberührt ließ. ${ }^{5}$

Bei einer Nachwahl wie in Dresden I kann eine Partei mit Überhangmandaten in dem betreffenden Bundesland ihren Anhängern gezielte Wahlempfehlungen für strategisches Wählen geben, die ein negatives Stimmgewicht zu ihrem Nachteil verhindern. Ein derart rationaler Umgang mit diesem Phänomen ist bei der Hauptwahl nicht ohne weiteres möglich, weil die Erwartungen des Wahlergebnisses in der Regel zu wenig präzise sein werden. Das negative Stimmgewicht bleibt aber auch dann ein Problem, wenn man es nicht auf Anhieb erkennen kann. Es verletzt einen Wahlgrundsatz, den man in der Social Choice Literatur das Monotonie-Prinzip nennt. ${ }^{6}$ Locker ausgedrückt darf der Wahlerfolg einer Partei nicht durch zusätzliche Stimmen vermindert werden. Entsprechend hat das Bundesverfassungsgericht den leichten Ausweg eines Nachwahlverbots, zum Beispiel durch die Aufstellung von Ersatzkandidaten für den Wahlkreisbewerber, nicht eröffnet. Seine Entscheidung lautet: „\$7 Absatz 2 Satz 3 in Verbindung mit $\$ 6$ Absätze 4 und 5 des Bundeswahlgesetzes (...) verletzt Artikel 38 Absatz 1 Satz 1 des Grundgesetzes, soweit hierdurch ermöglicht wird, dass ein Zuwachs an Zweitstimmen zu einem Verlust an Sitzen der Landeslisten oder ein Verlust an Zweitstimmen zu einem Zuwachs an Sitzen der Landeslis-

2 Vgl. inhaltlich Hans Meyer, Der Überhang und anderes Unterhaltsames aus Anlaß der Bundestagswahl 1994, in: KritV, 77. Jg. (1994), H. 4, S. $312-362$.

3 Vgl. Gerd Strohmeier, Vergangene und zukünftige Reformen des deutschen Wahlsystems, in: ders. (Hrsg.), Wahlsystemreform, Sonderband der ZPol, Baden-Baden 2009, S. $11-41$.

4 Hans Meyer, a.a.O. (Fn. 2), S. 321. Nach dem 1994 noch gültigen Hare / Niemeyer-System wurden die letzten auf eine Landesliste entfallenden Sitze nach dem größten Überrest im Sinn der höchsten Nachkommastellen verteilt. Meyer bezeichnet diese Mandate als Abrundungsmandate.

5 Vgl. Joachim Behnke, Strategisches Wählen bei der Nachwahl in Dresden zur Bundestagswahl 2005, in: PVS, 49. Jg. (2008), H. 4, S. 695 - 720; Gerd Strohmeier, a.a.O. (Fn. 3), S. 29 - 32.

6 Vgl. Kenneth Shepsle I Mark S. Bonchek, Analyzing Politics, New York 1997, S. 72 - 76; William H. Riker, Liberalism Against Populism, Prospect Heights 1982, S. $45-51$. 
ten führen kann."7 Inhaltlich wird dadurch ausgesagt, dass die Art der Unterverteilung der einer Partei nach der Oberverteilung auf Bundesebene zustehenden Sitze auf die verbundenen Landeslisten dieser Partei mit den Wahlgrundsätzen einer allgemeinen, unmittelbaren, freien, gleichen und geheimen Wahl nicht übereinstimmt, soweit es dadurch zum negativen Stimmgewicht kommen kann. Es werden damit nicht die Überhangmandate als solche verboten.

Es lohnt sich also der Frage nachzugehen, welche Möglichkeiten es für Überhangmandate ohne den bisherigen inversen Erfolgswert gibt. Man muss nicht gleich die weitergehende Lösung anstreben, Überhangmandate zu verhindern. Bevor sich eine Partei für die eine oder andere Lösung entscheidet, wird sie aber die Folgen einer Neuregelung für den eigenen Wahlerfolg prüfen. Diese Folgen sind nicht abstrakt zu bewerten, sondern unter dem gegenwärtig und in naher Zukunft existierenden Parteiensystem. Dementsprechend wird hier jeweils die Sitzverteilung berechnet, die die Neuregelungen mit den Ergebnissen der Wahl vom 27. September 2009 und für vorangegangene Wahlen erbracht hätten. Zwei solche möglichen Neuregelungen werden gegenübergestellt; bei beiden handelt es sich um „eine ,strukturerhaltend-korrigierende' Reform, das heißt eine Reform, die innerhalb der bestehenden personalisierten Verhältniswahl vollzogen wird und sich auf eine (...) ,Behebung' des Effekts des inversen Erfolgswerts konzentriert" ${ }^{\text {"8 }}$ Der erste Vorschlag beseitigt Überhangmandate weitgehend, indem die Zahl der erfolgreichen Direktkandidaten einer Partei bereits bei der Oberverteilung berücksichtigt wird. Diese Lösung entspricht dem Gesetzesvorschlag der Fraktion Bündnis 90/Die Grünen aus der letzten Wahlperiode, nach dem bereits der 17. Bundestag gewählt werden sollte. ${ }^{9}$ Diese Lösung wird hier als Bundeslösung bezeichnet und damit konkret die „direktmandatsbedingte Divisormethode mit Standardrundung (Augsburger Zuteilunsverfahren)“ von Pukelsheim gemeint. ${ }^{10}$

Der zweite Vorschlag, die so genannte Länderlösung, behandelt die Länder mit den entsprechenden Landeslisten der Parteien als geschlossene Wahlgebiete, in denen die Verteilung der Sitze nach Erst- und Zweitstimmen vorgenommen wird. Dieser Vorschlag ist verschiedentlich angesprochen worden, zum Beispiel vom Verfassungsgericht selbst oder von Strohmeier ${ }^{11}$, ausführlicher von Hahlen ${ }^{12}$ und Lübbert ${ }^{13}$ oder aus juristischer Perspektive von Meyer $^{14}$. Eine Auseinandersetzung aus sozialwissenschaftlicher Perspektive steht noch aus; so sind insbesondere seine Folgen für die Sitzverteilung noch zu prüfen. Eine solche Konkretisierung nehmen wir hier für diese Landeslösung vor, um auf diesem Weg zu Überhangmandaten ohne inversen Erfolgswert zu kommen. Erst daran anschließend werden die

7 BVerfG, a.a.O. (Fn. 1).

8 Gerd Strohmeier, a.a.O. (Fn. 3), S. 39.

9 Vgl. BT-Drs. 16/12855 vom 11. Februar 2009.

10 Vgl. Friedrich Pukelsheim, Bundeswahlgesetz - Nächste Etappe, in: DVB1., 123. Jg. (2008), H. 14, S. $889-897$.

11 Vgl. Gerd Strohmeier, a.a.O. (Fn. 3), S. 36.

12 Vgl. Johann Hahlen, Schriftliche Stellungnahme zur öffentlichen Anhörung des Innenausschusses des Deutschen Bundestages am 4. Mai 2009 in Berlin, Deutscher Bundestag 2009, A-Drs. 16 (4) 592 C.

13 Vgl. Daniel Lübbert, Negative Stimmgewichte und die Reform des Bundestags-Wahlrechts, in: Deutscher Bundestag - Wissenschaftliche Dienste 2009, WD 8-3000-020/09.

$14 \mathrm{Vgl}$. Hans Meyer, Lösungsmöglichkeiten nach dem Wahlrechtsurteil des BVerfG vom 3. Juli 2008, in: DVBl., 124. Jg. (2009), H. 3, S.137 - 146. 
Argumente geprüft, die für und gegen jeden der beiden Vorschläge sprechen. Dabei soll auch berücksichtigt werden, wie sich die neuen Regelungen wahrscheinlich auf die Wahlkampfstrategien der Parteien und die Motive der Bürger zum strategischen Wählen auswirken werden.

Die von Strohmeier ${ }^{15}$ sowie Franke und Grimmel ${ }^{16}$ vorgeschlagenen Varianten eines Grabenwahlsystems sind nicht mehr „strukturerhaltend-korrigierend“, sondern stellen größere Eingriffe in das bestehende Wahlrecht dar. ${ }^{17}$ Bei Beibehaltung der hälftigen Mandatsteilung zwischen Wahlkreis- und Listenmandaten würde eine Halbierung der Parlamentsstärke derjenigen Parteien folgen, die keine Wahlkreissiege erringen konnten. Im Prinzip wäre dies eine wohl nur von der Großen Koalition durchsetzbare Reform gewesen. Sie wird kaum von einer Regierung aus Union und FDP vorgeschlagen werden.

Im Folgenden wird für die Beibehaltung der Überhangmandate argumentiert und zur Vermeidung des inversen Erfolgswerts die Landeslösung vorgeschlagen. Überhangmandate sind eine Folge einer mit den Grundsätzen der Personenwahl (sprich: der relativen Mehrheitswahl in Einer-Wahlkreisen) verbundenen Verhältniswahl. Hauptargument wird sein, dass Beurteilungskriterien einer reinen Verhältniswahl für ein solches Mischwahlsystem unangemessen sind. Unsere wahlsystematisch begründete Konzeption eines Mischwahlsystems wird zu anderen Schlussfolgerungen führen als die juristisch aus den Wahlrechtsgrundsätzen von Art. 38 Abs. 1 Satz 1 GG abgeleiteten bisherigen Prüfkriterien des Bundesverfassungsgerichts.

\section{Auswirkungen von Bundes- und Landeslösung am Beispiel der Bundestagswahl 2009}

Friedrich Pukelsheim ${ }^{18}$ verspricht, dass mit dem Augsburger Zuteilungsverfahren Überhangmandate verhindert werden. Es wird bei der Unterverteilung der Sitze auf die Landeslisten einer Partei angewandt, nachdem bei der Oberverteilung auf Bundesebene zunächst die Parteistärken im neu gewählten Parlament nach der jetzt bereits praktizierten Methode Sainte-Laguë / Schepers auf Basis der Zweitstimmen berechnet worden sind. Dies ist ein Divisor-Verfahren, bei dem die Gesamtzahl der gültigen Zweitstimmen (ohne die Stimmen der Parteien, die an der Fünfprozenthürde beziehungsweise an der Grundmandateklausel gescheitert sind) durch die Zahl der zu vergebenden Sitze, also 598, geteilt wird. Das Ergebnis ist der Anfangsdivisor, der dann den Nenner abgibt für die gültigen Zweitstimmen der Parteien im Zähler. 2009 waren dies die Zweitstimmen der CDU als größter Partei mit fast zwölf Millionen, der SPD mit knapp zehn Millionen, der FDP mit 6,3, der Linken mit 5,2, der Grünen mit 4,6 und der CSU mit 2,8 Millionen Zweitstimmen. Mit Standardaufund -abrundung hätte die Summe der Mandate aber 600 ergeben. Deshalb musste der Zuteilungsdivisor leicht erhöht werden, um in der Summe auf 598 Mandate zu kommen,

15 Vgl. Gerd Strohmeier, Ein Plädoyer für die gemäßigte Mehrheitswahl. Optimale Lösung für Deutschland, Vorbild für Österreich und andere Demokratien, in: ZParl, 38. Jg. (2007), H. 2, S. $578-590$.

16 Vgl. Heiko Franke / Andreas Grimmel, Wahlen mit System? Reformüberlegungen zur personalisierten Verhältniswahl, in: ZParl, 38. Jg. (2007), H. 3, S. $591-602$.

17 Vgl. zu deren Auswirkungen Eric Linhart, Mögliche Auswirkungen von Grabenwahlsystemen in der Bundesrepublik Deutschland, in: ZParl, 40. Jg. (2009), H. 3, S. $624-647$.

18 Vgl. Friedrich Pukelsheim, a.a.O. (Fn. 10). 


\begin{tabular}{|l|r|r|c|c|c|c|}
\hline Tabelle 1: Die Zuteilung der Parteisitze an die verbundenen Landeslisten bei der Bundestagswabl \\
2009
\end{tabular}

davon 173 für die CDU, 146 für die SPD, 93 für die FDP, 76 für die Linke, 68 für die Grünen und 42 für die CSU. Die Verteilung dieser Sitze auf die Landeslisten der CDU als der am meisten betroffenen Partei (Unterverteilung) soll nun im Vergleich mit dem derzeit gültigen Verfahren für das Augsburger Zuteilungsverfahren berechnet werden.

Zum Vergleich wird die tatsächlich nach geltendem Bundestagswahlrecht vorgenommene Zuteilung der Parteisitze an alle verbundenen Landeslisten und die CSU herangezogen (vgl. Tabelle 1). Daraus geht auch die Zahl der Überhangmandate hervor, beziehungsweise genauer die Zahl der Direktmandate, die die nach der Zahl der Zweitstimmen zustehenden Proporzsitze übersteigen. ${ }^{19} 2009$ haben nur CDU und CSU von Überhangmandaten profitiert. Die CDU erhielt 21, davon allein zehn in Baden-Württemberg, vier in Sachsen, je zwei in Mecklenburg-Vorpommern und Rheinland-Pfalz und je eines in Schleswig-Holstein und Thüringen. Zum ersten Mal erhielt auch die CSU in Bayern Überhangmandate. Mit ihren 2,8 Millionen Zweitstimmen (6,5 Prozent auf Bundesebene) hätte sie 42 Sitze bekommen müssen. Da sie aber alle 45 Wahlkreise in Bayern gewonnen hat, stehen ihr 45 Sitze zu. Das heißt mit anderen Worten, dass kein einziger CSU-Abgeordneter über die Liste in den Bundestag einziehen konnte.

Genau diesen Effekt hätte die Bundeslösung nach dem Gesetzesvorschlag der Grünen auch für die CDU bei der letzten Bundestagswahl gehabt, wenn sie schon geltendes Recht

19 Vgl. zum Mandateüberhang Hans Meyer, a.a.O. (Fn. 2) oder Joachim Behnke, Das Wahlsystem der Bundesrepublik Deutschland, Baden-Baden 2007. 
gewesen wäre. Nach der Oberverteilung stehen der CDU 173 Sitze zu, und genau 173 ihrer Wahlkreiskandidaten waren erfolgreich. Ihre Verteilung auf die Länder steht damit fest, Zweitstimmenmandate würden nicht vergeben. Die Verschiebungen zwischen den Landesgruppen der CDU in der Bundestagsfraktion, die sich nach dem Augsburger Zuteilungsverfahren im Vergleich zur geltenden Unterverteilung ergeben, sind der Tabelle 2 zu entnehmen.

Generell ergeben sich starke Verschiebungen zugunsten der Parteien in Ländern mit Überhangmandaten, die von den Landesverbänden ohne Überhangmandate „bezahlt" werden müssten. So hätte die nordrhein-westfälische CDU nur noch Anspruch auf 37 statt auf 45 Mandate und die niedersächsische CDU auf 16 statt 21. Doch auch in Brandenburg würden der CDU vier Mandate verloren gehen, in Hamburg, Bremen, Sachsen-Anhalt und Berlin je eines. Trotz der 80.565 Zweitstimmen der CDU in Bremen wäre dieses Bundesland mit keinem einzigen CDU-Abgeordneten mehr im Bundestag vertreten. Die Bundestagsfraktion von CDU und CSU würde insgesamt süddeutscher, während die anderen Fraktionen ihre regionale Zusammensetzung behielten, weil sich ohne Überhangmandate an der Unterverteilung nichts ändert. Sie wird nach Sainte-Laguë vorgenommen, und die entsprechenden Parteidivisoren liegen mit Werten von 67.000 bis 69.000 auf der Höhe des Bundesdivisors für die Oberverteilung. Nur für die CDU würde sich ein Parteidivisor von 300.000 ergeben, um dem Land mit den wenigsten CDU-Zweitstimmen, nämlich Bremen, kein Mandat zuweisen zu müssen, da sonst die Obergrenze von 173 CDU-Sitzen nicht einzuhalten wäre. Ironischerweise führt die so genannte Bundeslösung für bei den Erststimmen besonders erfolgreiche Parteien zu mehr regionalen Ungleichheiten der Bundestagsfraktionen als das bisherige Wahlgesetz.

Das Augsburger Zuteilungsverfahren löst die Vorgabe des Bundesverfassungsgerichts, das negative Stimmgewicht zu beseitigen, durch die Aufteilung der Zweitstimmensitze auf die Landeslisten einer Partei erst nach Berücksichtigung der Direktmandate. 2005 hätten sich dann keine Überhangmandate ergeben. Dass sie nicht ganz abgeschafft wären, zeigt aber das Beispiel der CSU. Sie hat 2009 zum ersten Mal Überhangmandate gewonnen und zwar mit 48,2 Prozent der Erststimmen in Bayern und 42,6 Prozent der Zweitstimmen. Die Aussage des Erfinders des Verfahrens, es sei frei von Überhangmandaten ${ }^{20}$, war offensichtlich eine empirische Aussage, die auch falsch sein kann, und keine mathematisch-deduktive Aussage, die aus der Logik des Verfahrens abgeleitet worden wäre. Meyer ${ }^{21}$ hat in seiner Fassung der Bundeslösung dagegen mit der Möglichkeit von Überhangmandaten für die CSU gerechnet und vorgeschlagen, dass die CSU dann auf die Direktmandate „mit dem geringsten prozentualen Stimmgewicht" verzichten muss. Das wären 2009 die CSUAbgeordneten aus den Wahlkreisen München-Ost, München-Nord und Nürnberg-Süd gewesen, die ihr Direktmandat immerhin Erststimmenanteilen von 36,4 bis 36,6 Prozent verdanken und damit zehn Prozentpunkten mehr als die Bundestagsabgeordnete Eva Högl in Berlin-Mitte, die insgesamt mit dem niedrigsten Erststimmenanteil erfolgreich war. Der von Meyer so hoch gehaltene Gleichheitsgrundsatz gilt offensichtlich nur für die gültigen Zweitstimmen, bei denen, wie man sich immer erinnern sollte, ja auch bereits die Stimmen für die Parteien fehlen, die an der Fünfprozenthürde gescheitert sind. 


\begin{tabular}{|l|c|c|c|c|}
\hline Tabelle 2: Zuteilung der Sitze an CDU und CSU nach dem Augsburger Zuteilungsverfahren (1) \\
und dem 2009 geltenden Wablrecht (2)
\end{tabular}

Ist man an einer „strukturerhaltend-korrigierenden Reform“ nach Strohmeier ${ }^{22}$ interessiert, kann man die Überhangmandate bewusst und nicht nur als unschönen Nebeneffekt erhalten und nur den inversen Erfolgswert abschaffen. Dies gelingt mit unserer Landeslösung, die die Länder als geschlossene Wahlgebiete konstituieren würde. Die auf ein Land entfallenden Mandate werden vor der Wahl vom Gesetzgeber so fixiert, dass sie der anteiligen Aufteilung der Wahlberechtigten auf die Länder möglichst genau entsprechen. Der Einfachheit halber wird für die folgenden Analysen die doppelte Zahl der jetzigen Direktmandate pro Land verwendet. Mit der Bevölkerungsfortschreibung lassen sich natürlich vor jeder Wahl genauere Zahlen zugrunde legen, die auch leichte Abweichungen zwischen Direkt- und Zweitstimmenmandaten zuließen. Die Aufteilung dieser so oder so berechneten Mandate auf die Parteien in dem jeweiligen Land geschieht dann nach den für die jetzige Oberverteilung geltenden Regeln:

- An der Verteilung nach den Zweitstimmen nehmen die Parteien teil, auf die bundesweit mindestens fünf Prozent der Zweitstimmen entfallen sind oder die drei Direktmandate erhalten haben. ${ }^{23}$

- Die Zahl der einer Partei nach den Zweitstimmen zustehenden Sitze wird für jedes Land nach Sainte-Laguë berechnet.

22 Vgl. Gerd Strohmeier, a.a.O. (Fn. 3).

23 Ersatzweise ließe sich die Fünfprozentklausel auch auf die einzelnen Länder beschränken. 
- Die gewonnenen Direktmandate werden auf die Proporzquote angerechnet; über die Proporzquote hinausgehende Direktmandate bleiben erhalten.

Der Vollständigkeit halber muss erwähnt werden, dass die Stimmgebung nicht geändert wird. Jeder Wähler hat zwei Stimmen mit denselben Funktionen wie bisher. Er kann seiner Partei aber nicht mehr dadurch schaden, dass er ihr die Zweitstimme gibt. Mehr Stimmen führen zu einem gleichbleibenden oder besseren Endergebnis in dem jeweiligen Land, aber nie zu einem schlechteren Endergebnis.

Die Wahlkreiseinteilung wurde zuletzt für die Bundestagswahl 2009 geändert. Dabei erhielten Baden-Württemberg und Niedersachsen einen Wahlkreis mehr, während Sachsen und Sachsen-Anhalt einen Wahlkreis verloren. Wie aus Tabelle 3 hervorgeht, entspricht die Verteilung der Wahlkreise auf die Länder der Aufteilung der Wahlberechtigten sehr gut. Abweichungen von mehr als einem zehntel Prozentpunkt kommen nur zwei Mal vor und zwar vier zehntel Prozentpunkte zugunsten von Baden-Württemberg und drei zehntel Prozentpunkte zu Lasten von Sachsen.

Nach dem geltenden Wahlrecht wird die Abgeordnetenverteilung auf die Länder auch von der Wahlbeteiligung beeinflusst. So wurde als Ergebnis der Unterverteilung den Ländern Brandenburg, Berlin und Sachsen-Anhalt ein Sitz weniger zugeteilt, als der doppelten Anzahl der Wahlkreise entsprochen hätte. Mecklenburg, Sachsen und Thüringen konnten die Nachteile aus der geringeren Wahlbeteiligung durch Überhangmandate ausgleichen. Von den Ländern ohne Überhangmandate schnitt Hessen am besten bei der Sitzverteilung ab. Bei einer Spitzenwahlbeteiligung von 74 Prozent erhält Hessen drei Bundestagsabgeordnete mehr als die doppelte Wahlkreisquote.

\begin{tabular}{|l|c|c|c|c|}
\hline Tabelle 3: Verteilung der Wablberechtigten und der Direktmandate in Prozent auf die Länder \\
und doppelte Zabl der Direktmandate pro Land
\end{tabular}




\begin{tabular}{|c|c|c|c|c|c|c|c|c|}
\hline Land & & & SPD & FDP & Linke & Grüne & & \\
\hline Schleswig-Holstein & 9 & (2) & 6 & 4 & 2 & 3 & & \\
\hline Mecklenburg-Vorpommern & 6 & (1) & 3 & 1 & 4 & 1 & & \\
\hline Hamburg & 4 & & 3 & 2 & 1 & 2 & & \\
\hline Niedersachsen & 21 & & 19 & 8 & 5 & 7 & & \\
\hline Bremen & 1 & & (1) & 0 & 1 & 1 & & \\
\hline Brandenburg & 5 & & 6 & 2 & 6 & 1 & & \\
\hline Sachsen-Anhalt & 6 & & 3 & 2 & 6 & 1 & & \\
\hline Berlin & 6 & & 5 & 3 & 5 & 5 & & \\
\hline Nordrhein-Westfalen & 45 & & 38 & 20 & 11 & 14 & & \\
\hline Sachsen & 16 & (4) & 5 & 5 & 8 & 2 & & \\
\hline Hessen & 15 & (1) & 12 & 7 & 4 & 5 & & \\
\hline Thüringen & 7 & (1) & 3 & 2 & 6 & 1 & & \\
\hline Rheinland-Pfalz & 13 & (2) & 8 & 5 & 3 & 3 & & \\
\hline Bayern & & & 17 & 14 & 6 & 11 & 45 & (3) \\
\hline Baden-Württemberg & 37 & (9) & 16 & 15 & 6 & 11 & & \\
\hline Saarland & 4 & (2) & 2 & 1 & 2 & 1 & & \\
\hline Summe & 195 & $(22)$ & $148 \quad(1)$ & 91 & 76 & 69 & 45 & (3) \\
\hline \multicolumn{9}{|c|}{$\begin{array}{l}\text { Anmerkungen: In geschlossenen Wahlgebieten gilt Fünfprozentklausel bundesweit; Sitze in Klammerr } \\
\text { geben an, wie viele der angegebenen Mandate Überhangmandate sind. } \\
\text { Quelle: Eigene Berechnungen auf der Basis des Bundestagswahlergebnisses 2009, www.bundeswahlleiter } \\
\text { de/bundestagswahlen (Abruf im Dezember 2009). }\end{array}$} \\
\hline
\end{tabular}

Berechnet man nun die Sitzverteilung nach Sainte-Laguë / Schepers mit den Ländern als geschlossenen Wahlgebieten (vgl. Tabelle 4), muss zunächst betont werden, dass sich eine bundesweite Fünfprozenthürde auf das Wahlergebnis 2009 genauso auswirkt wie eine Fünfprozentklausel auf Landesebene. Jede der jetzt im Bundestag vertretenen Parteien gewann in jedem Bundesland mehr als fünf Prozent der Zweitstimmen, und keine sonstige Partei schaffte es, wenigstens in einem Land die Fünfprozenthürde zu überspringen. Der Logik der Länderlösung folgend könnte man überlegen, ob eine Fünfprozentklausel nicht besser auf Landesebene anzuwenden ist. Damit würde das Bundesergebnis ein rechtliches Nullum. Es verlöre an Wert für allfällige Berechnungen von nationalen Disproportionalitäten, die von den Anhängern der Verhältniswahl so geschätzt werden. Gegen eine Fünfprozentsperrklausel auf Landesebene spricht allerdings, dass sie die Repräsentation für kleine, aber dennoch bundesweit antretende Parteien erschwert. Stattdessen würde die Herausbildung starker unabhängiger Landesverbände angeregt. Um einer damit einher gehenden Schwächung des nationalen Parteiensystems vorzubeugen, erscheint es sinnvoll, die bisherige Sperrklausel auf Bundesebene beizubehalten.

Wie ein Blick auf die aus der Landeslösung resultierende Zusammensetzung des Bundestags zeigt, ist dieser Vorschlag in der Tat strukturerhaltend-korrigierend. Statt 194 Sitze bekäme die CDU 195 und davon 22 statt 21 Überhangmandate. Die SPD erhielte zwei Sitze mehr, nämlich 148 statt 146, davon ein Überhangmandat aus Bremen. Die FDP käme auf 91 statt 93, die Linke auf 76 wie bisher und die Grünen auf 69 statt 68 Abgeordnete. Die 45 Sitze der CSU blieben ebenfalls erhalten. Was die Länderaufteilung der CDUÜberhangmandate betrifft, änderte sich für Sachsen, Thüringen und Rheinland-Pfalz nichts 
im Vergleich zum geltenden System; Mecklenburg-Vorpommern und Baden-Württemberg verlören ein Mandat, und Schleswig-Holstein, Hessen und das Saarland gewönnen je ein zusätzliches Überhangmandat. Die entscheidende Frage ist jetzt, wie man eine Neuregelung des deutschen Wahlrechts einmal fast ohne Überhangmandate und einmal mit Überhangmandaten, aber ohne negatives Stimmgewicht, wahlsystematisch rechtfertigen kann.

\section{Wahlsystematische Beurteilung von möglichen Korrekturen des Bundestagswahlrechts}

Nach Grotz vereinigt „das deutsche Wahlsystem drei unterschiedliche Repräsentationsprinzipien (...): personalisiert-territoriale Repräsentation (in Form von Wahlkreismandaten), innerparteilich-föderalen Proporz (in Form von Landeslisten) und nationalen Parteienproporz (durch Errechnung der Gesamtmandate auf Bundesebene) “24. Das Augsburger Zuteilungsverfahren stärkt den nationalen Proporz auf Kosten des innerparteilich-föderalen Proporzes. Länder als geschlossene Wahlgebiete zu behandeln gibt den nationalen Parteienproporz als Hauptkriterium der Zusammensetzung des Bundestages auf und stärkt die personalisiert-territoriale Repräsentation. Beide Lösungen sind strukturerhaltend in dem Sinn, dass die wesentlichen Merkmale des gegenwärtigen Bundestagswahlrechts erhalten bleiben: die Kandidatenaufstellung, die Stimmgebung mit Erst- und Zweitstimme, das Prinzip der relativen Mehrheitswahl in Einer-Wahlkreisen, die Stimmverrechnung der Zweitstimmen nach Sainte-Laguë, die Vorrangstellung der Zweitstimme bei der Bestimmung der Sitzzahlen im Parlament beziehungsweise die Anrechnung von Wahlkreismandaten auf die Sitzzahlen der Parteien sowie, zumindest nach dem Gesetzesvorschlag der Grünen, die grundsätzliche Nicht-Aberkennung von einmal gewonnenen Direktmandaten. Letzteres spielt für die Bundeslösung eine untergeordnete Rolle, weil hier Überhangmandate gegenüber dem jetzigen Wahlrecht weitestgehend vermieden werden. Beide Lösungen sind insofern korrigierend, als sie das negative Stimmgewicht unmöglich machen.

Der Hauptunterschied von Bundes- und Landeslösung betrifft die wahlsystematische Interpretation des gegenwärtigen Wahlrechts. Danach entscheidet sich letztendlich, welche Eigenschaft, bei allen Gemeinsamkeiten der zwei Lösungen, erhalten bleiben soll. Die Bundeslösung setzt auf den „unitarischen Charakter des Bundestags. Der Wahlkörper bei einer Bundestagswahl ist das Bundesvolk und sind nicht die vereinigten Landesvölker" 25 . Diese petitio principii gewinnt erst dadurch Argumentationskraft, dass sie mit Prinzipien wie der Vermeidung von doppelten Stimmgewichten oder dem gleichen Erfolgswert der Stimmen verbunden wird. Mit ersterem Argument kann man sich auf das geltende Wahlrecht berufen. Es schließt doppelte Stimmgewichte in den - bisher nie aufgetretenen - Fällen aus, in denen ein Einzelbewerber ohne zugelassene Landesliste die Mehrheit der Erststimmen im Wahlkreis erhält. Die Zweitstimmen seiner Wähler werden dann nicht berücksichtigt, weil diese schon mit ihrer Erststimme einen Repräsentationserfolg erzielt haben. Die Wähler solcher Einzelkandidaten können somit bereits bei ihrer Stimmabgabe wissen, dass ihre Zweitstimmen eventuell nicht gezählt werden. Das unterscheidet diesen Fall von den so genannten Berliner Zweitstimmen. ${ }^{26}$ Dieses Phänomen war bei der Bundestagswahl 2002

24 Florian Grotz, Verhältniswahl und Regierbarkeit. Das deutsche Wahlsystem auf dem Prüfstand, in: Gerd Strohmeier (Hrsg.), a.a.O. (Fn. 3), S. 155 - 182, S. 173.

25 Hans Meyer, a.a.O. (Fn. 14), S. 141.

26 Vgl. dazu zum Beispiel Johann Hahlen, a.a.O. (Fn. 12). 
aufgetreten, als zwei Wahlkreiskandidaten der PDS in Berlin erfolgreich waren, ohne dass ihre Partei die Fünfprozenthürde überwunden oder ein dritter erfolgreicher Kandidat die Grundmandatsklausel erfüllt hätte. Für die PDS-Erststimmenwähler in den Wahlkreisen Berlin-Lichtenberg - Hohenschönhausen und Berlin-Marzahn - Hellersdorf war das bei der Stimmabgabe nicht offensichtlich.

Man kann das Argument des doppelten Stimmgewichts weiter strecken, indem man es auf das Stimmensplitting in Ländern mit erwartbaren Überhangmandaten ausdehnt, um so Überhangmandate generell als Verstoß gegen das Gleichheitsgebot erscheinen zu lassen. Meyer argumentiert so gegen die Landeslösung. „Hinzu kommt, dass das System in ,sicheren' Überhangländern wie Brandenburg (sic!), Sachsen, Sachsen-Anhalt (sic!) und wohl auch Baden-Württemberg den Anhängern der jeweilig entsprechenden großen Partei ermöglicht, sich ein doppeltes Stimmgewicht zu schaffen, indem sie ihre Zweitstimmen, die durch den zu erwartenden hohen Erfolg bei den Direktkandidaturen konsumiert würden, der nächst benachbarten Partei gäben." 27

Mit der Berufung auf das Gleichheitsgebot wird eine Verbindung zu einem zweiten Prinzip hergestellt, das häufig gegen Überhangmandate angeführt wird: dem Gebot eines gleichen Erfolgswerts der Wählerstimmen. Abweichungen vom bundesweit mit Hilfe der Zweitstimmen festgestellten Parteienproporz verletzten den gleichen Erfolgswert. Hier wird das Repräsentationsprinzip der Verhältniswahl zur Richtschnur genommen, allerdings, wie man hinzufügen möchte, einer reinen Verhältniswahl in einem nationalen Wahlkreis ohne Sperrklausel. Auch unter der gegenwärtigen - unter dem Gesichtspunkt der Erfolgswertgleichheit optimalen ${ }^{28}$ - Wahlregel nach Sainte-Laguë ${ }^{29}$ gilt Erfolgswertgleichheit nur im Hinblick auf Parteien, die die Sperrklausel überwinden. Überhangmandate sind im Vergleich dazu eine Petitesse.

Eine reduzierte Betrachtung des deutschen Wahlrechts allein unter dem Gesichtspunkt der Erfolgswertgleichheit erscheint verfehlt. Sie speist sich aus der vermutlich wahlhistorisch begründeten, unserer Ansicht nach aber fehlgeleiteten Sichtweise des deutschen Wahlrechts als reinem Verhältniswahlsystem. Das Plädoyer für die Erfolgswertgleichheit (mitsamt mathematischer Begründung) fußt auf der Prämisse, dass Wahlen lediglich die Funktion zukommt, eine Sitzverteilung im Parlament herzustellen, die mit der Stimmenverteilung übereinstimmt. Im deutschen Fall wird diese Stimmenverteilung dann noch ausschließlich mit der Zweitstimmenverteilung gleichgesetzt.

Die Neigung, das deutsche Wahlsystem als Verhältniswahl zu verstehen, ist weit verbreitet. Das kommt schon in der Bezeichnung „personalisierte Verhältniswahl“ zum Ausdruck, als handle es sich nur um eine besondere Form der Verhältniswahl. Das Bundeswahlgesetz selbst spricht in $\$ 1$ Abs. 1 von den „Grundsätzen einer mit der Personenwahl verbundenen Verhältniswahl“, um in Absatz 2 sofort zu konkretisieren: „Von den Abgeordneten werden 299 nach Kreiswahlvorschlägen in den Wahlkreisen und die übrigen nach Landesvorschlä-

27 Hans Meyer, a.a.O. (Fn. 14), S. 141.

28 Erfolgswertgleichheit bedeutet, dass die Vertretungszahl (Stimmen pro Parteisitz) jeder Partei möglichst wenig von der theoretischen Vertretungszahl (Gesamtstimmen geteilt durch Sitze) abweichen soll. Sainte-Laguë ist das Verfahren, das die quadratischen Abweichungen der Vertretungszahlen minimiert. Erfolgswertgleichheit ließe sich aber auch herstellen, indem man die absoluten Abweichungen der Vertretungszahlen minimiert. Allerdings wäre Sainte-Laguë dann nicht mehr jedem anderen Verfahren vorzuziehen.

29 Vgl. Friedrich Pukelsheim, a.a.O. (Fn. 10), S. 892. 
gen (Landeslisten) gewählt." Damit ist die Personenwahl auf eine Mehrheitswahl in EinerWahlkreisen festgelegt. Weil die Wirkung dieses Wahlsystems lange Zeit Bundestage mit einer Parteizusammensetzung zur Folge hatte, die nur geringe Abweichungen von der Zweitstimmenverteilung aufwies, schien allein von der Repräsentationsfunktion der Wahl her betrachtet die Einordnung des deutschen Wahlsystems als eine Unterart der Verhältniswahl gerechtfertigt. ${ }^{30}$ Wenn in diesem System Abweichungen zwischen Wähler- und Abgeordnetenverteilung auftreten, sind sie in erster Linie der Fünfprozenthürde geschuldet und nicht der Mehrheitswahl in Einer-Wahlkreisen. ${ }^{31}$

Man kann ein Wahlsystem aber auch nach anderen Kriterien beurteilen als nach seinen Auswirkungen auf die Disproportionalität zwischen Stimmen- und Sitzverteilung. In der englischsprachigen Literatur wird das deutsche System als „mixed-member proportional“ bezeichnet. ${ }^{32}$ Damit wird auf die Tatsache rekurriert, dass ein Teil der Abgeordneten namentlich in Wahlkreisen und ein anderer Teil der Abgeordneten desselben Parlaments als Listenkandidaten in einem größeren, überlappenden Wahlkreis gewählt werden. Die Abgeordneten des Bundestags sind politikwissenschaftlich gesehen verschieden legitimiert, eine Hälfte verdankt ihr Mandat der Delegierten- oder Mitgliederversammlung in den Wahlkreisen und einer relativen Mehrheit der dortigen Wähler, die andere Hälfte einer Landesdelegiertenversammlung und dem Zweitstimmenanteil der jeweiligen Partei. Der Direktkandidat muss lokale Mehrheiten von sich überzeugen. Ob er selbst oder seine Partei hier die größere Überzeugungskraft aufbringt, ist nicht entscheidend. Wichtig ist die Abhängigkeit von lokalen Wählermehrheiten. Extremistische Kandidaten, deren Parteien es bundesweit auf über fünf Prozent bringen könnten, dürften nicht ohne weiteres in der Lage sein, auch relative Wählermehrheiten in Wahlkreisen von sich oder ihrer Partei zu überzeugen.

Fasst man das deutsche Wahlsystem als Mischwahlsystem in diesem Sinn auf, stellen Überhangmandate kein Problem dar. Die Fiktion vom Repräsentationsziel der reinen Verhältniswahl und daraus abgeleitete Prinzipien wie Erfolgswertgleichheit sind damit als Beurteilungskriterium sekundär. Jedes der zwei Segmente des Parlaments ist vom Wähler legitimiert, wenn auch auf unterschiedliche Weise, und für jedes Segment steht dem Wähler eine Stimme zur Verfügung, die Erststimme für den Wahlkreiskandidaten und die Zweitstimme für die Landesliste einer Partei. Die Verbindung zwischen Personen- und Verhältniswahl kommt durch die Anrechnung der siegreichen Wahlkreiskandidaten einer Partei auf die ihr zustehenden Listenmandate zum Ausdruck. Dadurch ergab sich bisher ein fast vollständig kompensatorisches Mischwahlsystem zugunsten der Verhältniswahl. Der Mischwahlcharakter in der Legitimationskette wird dadurch aber nicht aufgehoben.

In diesem Mischwahlsystem stellen Überhangmandate einen möglichen Mehrheitsbonus für die Partei dar, die sich in einem Bundesland flächendeckend in den Wahlkreisen als mehrheitsfähig erwiesen hat. Mehrheitsboni werden zur Erleichterung der Regierungsbil-

30 So Dieter Nohlen, Erfolgswertgleichheit als fixe Idee oder: Zurück zu Weimar? Zum Urteil des Bundesverfassungsgerichts über das Bundeswahlgesetz vom 3. Juli 2008, in: ZParl, 40. Jg. (2009), H. 1, S. $179-195$.

31 Vgl. Eric Linhart, a.a.O. (Fn. 17).

32 Vgl. zum Beispiel Matthew S. Shugart / Martin P. Wattenberg, Mixed-Member Electoral Systems: A Definition and Typology, in: dies. (Hrsg.), Mixed-Member Electoral Systems. The Best of Both Worlds?, Oxford 2001, S. $9-24$. 
dung verschiedentlich eingesetzt, so im griechischen und im italienischen Wahlsystem. Ein mehrheitsbildender Effekt nach deutschem Wahlrecht muss nicht bei jeder Wahl auftreten. Eine Ursache dieser Fähigkeit, Direktkandidaten durchzubringen, ist das Stimmensplitting. Einer lokal mehrheitsfähigen Partei gibt man die Erststimme und einer kleineren Partei die Zweitstimme. Ein solches Verhalten bietet sich als Form des Koalitionswählens an. Wenn die entsprechenden Parteien Koalitionsabsichten signalisieren und wenn der Wähler eine solche Koalition für im Prinzip mehrheitsfähig hält, kann er beide Koalitionsparteien wählen und auf diese Weise einen möglichen Mehrheitsbonus zugunsten des Parteilagers erzeugen, das sich besser koordinieren kann. ${ }^{33}$ Die Parteien müssen also vor der Wahl klare Koalitionsoptionen anbieten und Wähler, die eine bestimmte Regierungskoalition unterstützen, müssen in großer Zahl ihre Stimmen nach einem einheitlichen Muster splitten.

Um die Wirkungsweise von Überhangmandaten als Mehrheitsbonus zu verdeutlichen, sind in den Tabellen 5 bis 7 die Sitzverteilungen aufgeführt, die sich in den letzten drei Bundestagswahlen unter der Landeslösung ergeben hätten. Betrachtet man die Ergebnisse in den Tabellen, so fällt zunächst auf, dass die Landeslösung weniger Überhangmandate produziert als die damals angewandten Verfahren. ${ }^{34}$ Das liegt daran, dass unter der länderweisen Sitzzuteilung bestimmte Ursachen von Überhangmandaten wie ungleiche Wahlbeteiligung entfallen. Als wesentliche Ursache verbleibt das Stimmensplitting. So ergibt sich, dass die SPD 1998 und 2002 als einzige Partei Überhangmandate erhält, während 2005 SPD und CDU etwa gleich viele Überhangmandate erzielen.

Man könnte argumentieren, dass es drei Typen von Wahlen gibt: Die Wahl einer neuen Regierungsmehrheit ins Amt, die Wiederwahl einer Regierungsmehrheit und die misslungene Koordination, also keine klare Mandatierung einer Mehrheit. Als Beispiel für den ersten Typus dienen die Bundestagswahlen 1998 und 2009 (vgl. Tabelle 4). In diesen Wahlen fallen Überhangmandate ausschließlich für den Sieger an. 2002 kann als Beispiel für eine Wiederwahl interpretiert werden, in der Überhangmandate der amtierenden Regierungsmehrheit einen Mehrheitsbonus verschaffen (man beachte, dass die SPD unter dem vorgeschlagenen Verfahren nicht, wie damals, lediglich um Haaresbreite zur größten Fraktion geworden wäre). Die Wahl 2005 könnte man schließlich als ein Beispiel misslungener Koordination deuten. Weder Regierungs- noch Oppositionslager konnten eine Mehrheit der Wähler von sich überzeugen; entsprechend erhielten beide unter dem vorgeschlagenen Verfahren etwa gleich viele Überhangmandate, und ein mehrheitsbildender Effekt blieb aus.

Diese Überlegungen zeigen, dass ein Mehrheitsbonus durch Überhangmandate keineswegs garantiert ist, sondern nur dann auftreten sollte, wenn Parteien sich erstens vor der Wahl zu klaren Koalitionen bekennen und zweitens diese Koalitionsaussagen eine ausreichend große Zahl von Wählern dazu veranlassen, ihre Stimmen in identischer Weise zu splitten. Im unrealistischen Grenzfall könnten alle Anhänger einer Koalition ihre Stimmen so splitten, dass sie alle dieselbe Partei mit der Zweitstimme und Kandidaten der anderen Partei mit der Erststimme wählen, so dass diese Partei nur mit Überhangmandaten in den

33 Vgl. Franz Urban Pappi / Alexander Herzog / Ralf Schmitt, Koalitionssignale und die Kombination von Erst- und Zweitstimme bei den Bundestagswahlen 1953 bis 2005, in: ZParl, 37. Jg. (2006), H. 3, S. 493 - 513.

34 Aus Platzgründen wird auf die Angabe der damaligen Wahlergebnisse verzichtet. 


\begin{tabular}{|l|c|c|c|c|c|c|}
\hline Tabelle 5: Sitzverteilung nach Sainte-Laguë / Schepers mit Ländern als geschlossene Wablgebiete \\
auf der Basis des Bundestagswahlergebnisses 1998
\end{tabular}

\begin{tabular}{|c|c|c|c|c|c|c|}
\hline \multicolumn{7}{|c|}{$\begin{array}{l}\text { Tabelle 6: Sitzverteilung nach Sainte-Laguë / Schepers mit Ländern als geschlossene Wablgebiete } \\
\text { auf der Basis des Bundestagswahlergebnisses } 2002\end{array}$} \\
\hline Land & $\mathrm{CDU}$ & SPD & FDP & Linke & Grüne & CSU \\
\hline Schleswig-Holstein & 8 & 10 & 2 & 0 & 2 & \\
\hline Mecklenburg-Vorpommern & 5 & 7 & 1 & 0 & 1 & \\
\hline Hamburg & 4 & (1) & 1 & 0 & 2 & \\
\hline Niedersachsen & 21 & 29 & 4 & 0 & 4 & \\
\hline Bremen & 1 & 2 & 0 & 0 & 1 & \\
\hline Brandenburg & 6 & 12 & 1 & 0 & 1 & \\
\hline Sachsen-Anhalt & 7 & 10 & 2 & 0 & 1 & \\
\hline Berlin & 7 & (2) & 2 & 2 & 4 & \\
\hline Nordrhein-Westfalen & 47 & 57 & 12 & 0 & 12 & \\
\hline Sachsen & 15 & 14 & 3 & 0 & 2 & \\
\hline Hessen & 16 & 17 & 4 & 0 & 5 & \\
\hline Thüringen & 8 & 10 & 1 & 0 & 1 & \\
\hline Rheinland-Pfalz & 13 & 12 & 3 & 0 & 2 & \\
\hline Bayern & & 30 & 5 & 0 & 9 & 44 \\
\hline Baden-Württemberg & 33 & 26 & 6 & 0 & 9 & \\
\hline Saarland & 3 & 4 & 0 & 0 & 1 & \\
\hline Summe & 194 & $257 \quad(3)$ & 47 & 2 & 57 & 44 \\
\hline \multicolumn{7}{|c|}{$\begin{array}{l}\text { Anmerkungen: In geschlossenen Wahlgebieten gilt die Fünfprozentklausel bundesweit; Sitze in Klam- } \\
\text { mern geben an, wie viele der Mandate Überhangmandate sind. } \\
\text { Quelle: Eigene Berechnungen auf der Basis des Bundestagswahlergebnisses 2002, www.bundeswahlleiter. } \\
\text { de/bundestagswahlen (Abruf im Dezember 2009) }\end{array}$} \\
\hline
\end{tabular}




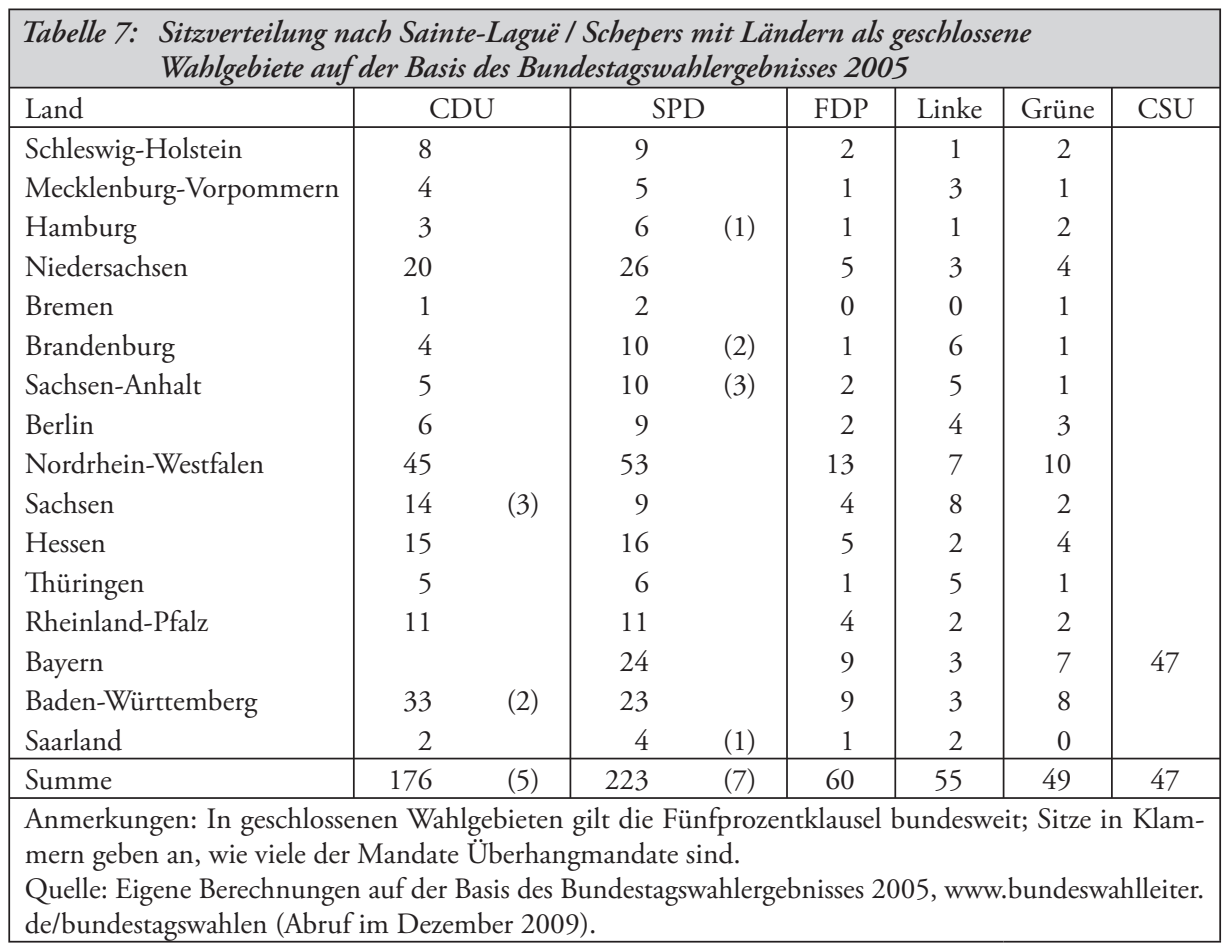

Bundestag einzieht. ${ }^{35}$ Solange die Parteien aber Nachteile bei der Zweitstimme befürchten, wenn sie nicht flächendeckend Wahlkreiskandidaten aufstellen ${ }^{36}$, und solange Parteiwählen das Koalitionswählen überwiegt, wird der Grenzfall nicht eintreten. ${ }^{37}$ Im Gedankenexperiment demonstriert er aber gut, dass man auch mit diesem Mischwahlsystem alternierende Regierungsmehrheiten zustande bringen könnte.

Neben der Entstehung von Überhangmandaten ist auch wichtig, ob eine länderweise Sitzzuteilung kleinere Parteien benachteiligen würde. A priori wäre diese Frage zu bejaen, denn statt bisher 598 Sitze stünden, je nach Bundesland, nur noch zwischen 128 (Nordrhein-Westfalen) und vier Sitze (Bremen) zur Vergabe über die Zweitstimme zur Verfügung, und jede Verkleinerung der Zahl zu vergebender Sitze schmälert theoretisch die Chancen kleinerer Parteien, einen Sitz zu erringen. Ein Blick auf die Sitzverteilungen in Tabellen 4

35 Vgl. Ossip Fürnberg / Danko Knothe, Wahlsieg ohne Stimmenmehrheit: Auswirkungen von verstärktem „Lagersplitting“ auf Mandatsverteilung und Koalitionsoptionen, in: ZParl, 40. Jg. (2009), H. 1, S. $56-74$.

36 Vgl. Susumu Shikano, Simulating Party Competition and Vote Decision Under Mixed Member Electoral Systems, in: AUCO Czech Economic Review, 3. Jg. (2009), H. 3, S. 270 - 291.

37 Auch der von Fürnberg und Knothe (Fn. 35) beschriebene Effekt, dass Koalitionswählen zu unintendierten Mehrheiten über Lagergrenzen hinweg führen kann, ist unwahrscheinlich, solange bei den Anhängern großer Parteien die Parteiwahl die Koalitionswahl überwiegt. Zudem ließe sich der Effekt abschwächen, indem Koalitionswähler nur in Ländern, in denen eine große Zahl von Wahlkreisgewinnen für eine der Koalitionsparteien erwartet wird, ihre Zweitstimme jeweils der anderen Koalitionspartei geben, in allen anderen Ländern ihre Zweitstimme dagegen der jeweils präferierten Koalitionspartei geben. 
bis 7 zeigt indes, dass sowohl FDP, Grüne als auch Linke unter der Landeslösung praktisch dieselben Ergebnisse erzielen würden wie unter dem gegenwärtigen Verfahren. Das liegt vor allem an der bundesweiten Fünfprozenthürde, die im Durchschnitt etwa dieselbe Wirkung entfaltet wie eine Einteilung des gesamten Bundesgebietes in gleich große Wahlbezirke mit je 14 Mandaten. ${ }^{38}$ Nur zwei Länder liegen bei dem vorgeschlagenen Verfahren unterhalb dieser theoretischen Schwelle: Bremen mit insgesamt vier und das Saarland mit zehn zu vergebenden Sitzen. Nur dort sollte es für kleinere Parteien im Durchschnitt schwerer werden, Sitze zu erringen. In allen anderen Ländern wäre, wie bisher auch, die bundesweite Fünfprozentsperrklausel und nicht die Mandatszahl die eigentliche Einstiegshürde für kleinere Parteien. Erhebliche Verzerrungen in den Vertretungszahlen kleiner Parteien sind daher nicht zu erwarten.

Für eine vollständige Beurteilung der hier vorgeschlagenen Landeslösung - Überhangmandate ohne negatives Stimmgewicht - muss noch bedacht werden, wie sie sich ihrerseits wahrscheinlich auf das Parteiensystem auswirken wird. Jede Wahlrechtsreform kann Anreize für eine Änderung des bisherigen Partei- und Wählerverhaltens bieten, so dass es nicht ausreicht, nur ein unter dem bisherigen Wahlrecht zustande gekommenes Wahlergebnis auf das neue Wahlrecht mechanisch umzurechnen.

\section{Auswirkungen von Wahlrechtskorrekturen auf das Parteiensystem}

Das Wahlrecht ist keine neutrale Institution, die vorhandene Wählerpräferenzen in die Stimmgebung und die Berechnung des Endergebnisses transformiert, sondern es beeinflusst auch die Art und Weise, wie Bürger ihre Präferenzen in Wahlentscheidungen umsetzen, wie Parteien Kandidaten aufstellen und die Wähler zu beeinflussen versuchen. Die hier diskutierten Bundes- und Landeslösungen zur Vermeidung eines negativen Stimmgewichts ändern an der Stimmgebung nichts, so dass die Wähler zunächst keinen Anlass haben, unmittelbar zu reagieren. Das kann sich aber schnell unter dem Einfluss von Parteiempfehlungen für die Wahlentscheidung ändern.

Die Bundeslösung wird so, wie sie im Gesetzentwurf der Grünen vorgesehen war, bei den Parteien, die in einzelnen Ländern Überhangmandate erwarten können, die Überlegung auslösen, ob sie nicht den Austritt aus dem Verbund ihrer Landeslisten vornehmen sollen. Dann müssen wie bei der CSU nicht andere Landeslisten für ihre Überhangmandate bezahlen. Derartige strategische Entscheidungen können nur durch einen vom Gesetz ausdrücklich ausgesprochenen Zwangsverband verhindert werden. ${ }^{39}$ Soweit mit der Bun-

38 Arend Lijphart (Patterns of Democracy, London u.a. 1999, S. 153) gibt eine einfache Beziehung zwischen Sperrklausel und Wahlkreiszuschnitt an, nach der eine Einteilung des Wahlgebiets in gleiche Wahlbezirke mit jeweils $\mathrm{M}$ zu vergebenden Mandaten im Durchschnitt eine ähnlich hohe Hürde darstellt, wie eine bundesweite Stimmverrechnung mit einer Sperrklausel von 75/(M+1) Prozent. Für eine theoretische Begründung dieser Beziehung, siehe Lijphart, Electoral Systems and Party Systems, New York 1994, S. 25 ff. und 182 f. Für eine generelle Übersicht, welche Sperrklauseln durch Mandatszahl, Stimmenverrechnungsformel und Anzahl der Parteien im Wahlkreis implizit gesetzt werden, siehe Michael Gallagher, Proportional Representation Electoral Systems: Quotas, Thresholds, Paradoxes and Majorities, in: British Journal of Political Science, 22. Jg. (1992), H. 4, S. 56 - 74, insbesondere Abbildung 2.

39 Vgl. Johann Hahlen, a.a.O. (Fn. 12). 
deslösung Überhangmandate tatsächlich verhindert werden, wird dem Stimmensplitting die koalitionsfördernde Wirkung entzogen. Es bleibt dann nur ein expressives Koalitionswählen, das jenen Parteien schadet, die „nur" die Erststimme bekommen. Das sind die Parteien, die bisher mehr Erst- als Zweitstimmen erhalten haben, im Wesentlichen also CDU, CSU und SPD. Diese Parteien dürften sich massiver als bisher gegen ein Stimmensplitting aussprechen, was sich, im Falle einer realistisch ins Auge gefassten Regierungswechsel-Koalition, durchaus zu Lasten des kleineren Koalitionspartners auswirken könnte. Die großen Parteien würden damit aber letztlich nur versuchen zu erreichen, worauf sie sich als Große Koalition nicht zu ihrem beiderseitigen Vorteil per Gesetz geeinigt haben, nämlich eine Wahlentscheidung für dieselbe Partei bei Erst- und Zweitstimme: Durch eine Wahlgesetzänderung hätten CDU/CDU und SPD dem Wähler - wie bei der Bundestagswahl 1949 - nur eine Stimme geben können, die dann doppelt ausgezählt wird - zur Ermittlung des Wahlkreissiegers und zur Ermittlung des Parteienproporzes. ${ }^{40}$

Die Bundeslösung würde das bisherige Parteiensystem auch insofern tangieren, als der innerparteiliche Proporz bei den großen Parteien gestört würde. ${ }^{41}$ Der frühere Überhangbonus einzelner Landesverbände wird zu einem merklichen Malus für jene Landesverbände, die für die Mandate bezahlen müssten, die nach geltendem Wahlrecht Überhänge schaffen.

Als Nachteil der Landeslösung diagnostiziert Meyer ${ }^{42}$ eine Schwächung des Zentralstaats und die Stärkung zentrifugaler Kräfte im Parteiensystem. Dabei sollte man nicht übersehen, dass auch nach geltendem Recht und der Bundeslösung die Kandidatenaufstellung den Wahlkreis- und Landesverbänden vorbehalten bleibt. Die Bundespartei stellt keine Kandidaten auf. Dass in der alten Bundesrepublik nach 1949 ein funktionierendes Parteiensystem auf Bundesebene entstanden ist, ist der Integrationskraft der Parteien, der Fünfprozentsperrklausel auf Bundesebene und den föderalen Gesetzeskompetenzen, vor allem auch des Bundesrats, zu verdanken. Erst durch die deutsche Einheit kam es wieder zu größeren regionalen Unterschieden im Parteiensystem, die bisher auch von einer Bundeslösung nicht aufgehoben wurden.

Hinsichtlich der mittel- und langfristig zu erwartenden Wirkungen der hier vorgeschlagenen Landeslösung ist Folgendes festzuhalten. Über die Beibehaltung der Möglichkeit von Überhangmandaten bleibt auch der Mehrheitsbonus möglich, der die Regierungsbildung erleichtern kann. Dieser Mehrheitsbonus kommt nur Parteien zugute, die flächendeckend mehrheitsfähig sind. Damit ist gemeint, dass diese Parteien in größerem Umfang Direktkandidaten bei der Bundeswahl durchbringen können. In Westdeutschland hat sich nach 1953, als das Zweitstimmenverfahren eingeführt wurde, sehr schnell ein Zweiparteiensystem auf Wahlkreisebene in dem Sinn etabliert, dass CDU beziehungsweise CSU und SPD die stärkste und zweitstärkste Partei bei den Erststimmen stellen.

Das Gesetz von Duverger, wonach Mehrheitswahl in Einer-Wahlkreisen stets zu einem Zweiparteiensystem führt ${ }^{43}$, hat sich im Mischwahlsystem auf Wahlkreisebene in Deutsch-

40 Negative Stimmgewichte ließen sich durch diese Änderung allein allerdings nicht vermeiden.

41 Vgl. dazu auch Johann Hablen, a.a.O. (Fn. 12).

42 Vgl. Hans Meyer, a.a.O. (Fn. 14).

43 Vgl. Maurice Duverger, Duverger's Law. Forty Years Later, in: Bernard Grofman / Arend Lijphart (Hrsg.), Electoral Laws and Their Political Consequences, New York 1994, S. 69 - 84; Gary W. Cox, Making Votes Count, Cambridge 1997. 
land besser bewährt als in Großbritannien. Shikano ${ }^{44}$ erklärt dies damit, dass sich die deutschen Wähler stark an den zu erwartenden nationalen Ergebnissen orientieren. Allerdings funktioniert dies nur in den alten Bundesländern. In den ostdeutschen Ländern hat sich seit der Wiedervereinigung hinsichtlich der Mehrheitsfähigkeit ein Dreiparteiensystem etabliert mit der PDS und später der Linken als einer weiteren großen Partei. Dabei war 2009, im Gegensatz zu früheren Wahlen, die SPD beim Erststimmenergebnis in MecklenburgVorpommern, Sachsen-Anhalt, Sachsen und Thüringen in der Regel nur noch die drittstärkste Partei; das galt für die CDU in Brandenburg. Die Dreiparteienkonstellation in den neuen Bundesländern und die damit einhergehenden knappen Erststimmenabstände zwischen erst-, zweit- und drittplatzierten Kandidaten machen es für den Wähler schwierig, die wahrscheinliche Parteistärke abzuschätzen.

Aufgrund der Unsicherheit über die Chancen der Kandidaten im Wahlkreis kann man für die ostdeutschen Länder zurzeit eher von einer Abwesenheit Duvergerscher Zwei-Parteien-Gleichgewichte ${ }^{45}$ ausgehen, unter der mehrheitsbildende Effekte für eine SPD-geführte Koalition nur schwer herbeizuführen sind. Da die Landeslösung aber derjenigen elektoralen Koalition einen Mehrheitsbonus verschafft, die sich und ihre Wähler besser koordinieren kann, wäre mittelfristig zu erwarten, dass die SPD entweder versucht, in den neuen Ländern wieder mindestens zweitstärkste Kraft zu werden, oder dass sie sich auf die Linke zubewegt, um vorhandene Koordinationspotentiale bei der Aufstellung von Wahlkreiskandidaten auszuloten.

Ungeachtet der soeben skizzierten Möglichkeiten strategischer Koordination sei abschließend angemerkt, dass nicht davon ausgegangen werden kann, dass Parteien- und Wählerverhalten unter der dargestellten Landeslösung erheblich von dem abweichen werden, was gegenwärtig zu beobachten ist. Das geltende Wahlrecht setzt bereits Anreize zur Koordination und zur Erzeugung von Überhangmandaten, dennoch konzentriert sich der Parteienwettbewerb in erster Linie auf die Gewinnung der Zweitstimme. Trotz der mehrheitsbildenden Elemente im deutschen Mischwahlsystem kann von einem Wettlauf um Überhangmandate keine Rede sein.

\section{Schlussbetrachtung: Das Beste aus Mehrheits- und Verhältniswahl}

Mit dem hier vorgeschlagenen und berechneten Sitzzuteilungsverfahren, das sich von der gegenwärtigen Praxis nur darin unterscheidet, dass alle Mandate auf Landesebene vergeben werden, würde die Möglichkeit eines negativen Stimmgewichts eliminiert, während die wesentlichen Strukturmerkmale des geltenden Bundestagswahlrechts (Kandidatenaufstellung, Stimmgebung, Verrechnung von Erst- und Zweitstimme und insbesondere die Möglichkeit von Überhangmandaten) allesamt erhalten blieben.

Andere in der Literatur diskutierte Vorschläge zur Beseitigung eines negativen Stimmgewichts hätten nicht dieselben strukturerhaltenden Wirkungen: Die Bundeslösung des Augsburger Zuteilungsverfahrens etwa macht die Einhaltung des Landesproporzes vom Wahlergebnis abhängig und setzt damit Anreize für Landeslisten, aus dem Bundesverband auszutreten. Hinzu kommt, dass der Austritt aus dem Verbund Überhangmandate ermög- 
licht, die im Verbund nicht anfallen würden. Auch andere, in diesem Beitrag nicht diskutierte Vorschläge hätten nicht dieselben strukturerhaltend korrigierenden Wirkungen: Das häufig zitierte Grabenwahlsystem ohne Verrechnung von Direkt- und Listenmandaten würde, wie auch das einfache Mehrheitswahlsystem, zu Wahlergebnissen führen, die weitab von dem liegen, was Wähler und Parteien in Deutschland gewohnt sind. Ein reines Verhältniswahlsystem mit Fünfprozentklausel würde die Proportionalität des Wahlergebnisses nicht wesentlich verbessern, und selbst wenn man offene Listen zuließe ${ }^{46}$, wäre dies auf Landesebene nur ein schwacher Ersatz für die jetzige Personenwahl in Einer-Wahlkreisen.

Die einzigartige Struktur des deutschen Wahlsystems hat sich über einen langen Zeitraum bewährt: Das Mischwahlsystem ermöglichte stabile, zumindest 1998 sogar alternierende Regierungsmehrheiten bei gleichzeitiger Wahrung von Parteien- und Landesproporz; es sorgte einerseits für die Entstehung geschlossener Parteiverbände mit nationaler Ausrichtung, ohne jedoch andererseits auf volksnahe, direkt gewählte Abgeordnete zu verzichten. Die unterschiedlichen Auswirkungen der Stimmabgabe in beiden Wahlgängen und die Gelegenheit zur individuellen Stimmgebung bieten dem Wähler Möglichkeiten, wie sie kaum ein anderes Wahlsystem bereithält. Seit Ende der achtziger Jahre hat sich das Mischwahlsystem deutscher Prägung zu einem wahren Exportschlager entwickelt. Es ist das meistadoptierte System der jüngeren Vergangenheit, und es vereint nach Ansicht vieler Experten das Beste aus den Welten von Mehrheits- und Verhältniswahl. ${ }^{47}$

Bei der anstehenden Reform sollte es deshalb vor allem darum gehen, das deutsche Wahlsystem in seiner bisherigen Form zu bewahren. Der Fehler eines möglichen inversen Erfolgswerts von Wählerstimmen ließe sich mit der hier vorgestellten länderweisen Sitzzuteilung beheben, ohne damit das deutsche Wahlsystem in seiner Wirkungsweise grundlegend zu verändern.

46 Vgl. den Vorschlag von Eric Linhart, a.a.O. (Fn. 17).

47 Vgl. Matthew S. Shugart / Martin P. Wattenberg, a.a.O. (Fn. 32).

\section{Negative Stimmgewichte bei der Bundestagswahl 2009*}

\section{Daniel Lübbert}

Aufgrund der bei der Bundestagswahl 2005 nötig gewordenen Nachwahl in Dresden ist einer breiteren Öffentlichkeit erstmals bewusst geworden, was eine kleine Gruppe von Experten schon früher bemerkt hatte: Unter bestimmten Umständen kann das geltende Wahlsystem dazu führen, dass zusätzliche Wählerstimmen einer Partei nicht nützen, sondern schaden. ${ }^{1}$ Grund dafür ist eine komplexe Wechselwirkung zwischen den Auswirkun-

* Der Autor ist Mitarbeiter der Wissenschaftlichen Dienste des Deutschen Bundestages. Der Beitrag gibt ausschließlich seine persönliche Auffassung wieder.

1 Vgl. Gerald Mann, Die unumgängliche Umkehr bei der Berechnung von Überhangmandaten: Reformvorschläge, in: ZParl, 27. Jg. (1996), H. 3, S. 398 - 404. 